\title{
Die OSZE und der Kampf gegen den Antisemitismus in Europa
}

\author{
Rabbi Andrew Baker"
}

\section{Zusammenfassung}

Dieser Beitrag untersucht den Kampf der OSZE gegen den Antisemitismus und unterstreicht die Sicherheitsbedenken jüdischer Gemeinden und die Bedeutung einer gemeinsamen Definition von Antisemitismus. Ausgehend von den Erfahrungen des Autors in seiner Rolle als Persönlicher Beauftragter des Amtierenden Vorsitzenden der OSZE für die Bekämpfung des Antisemitismus beleuchtet der Beitrag auch Herausforderungen, die sich aus der Organisationsstruktur, der Finanzierung und den Entscheidungsprozessen der OSZE ergeben, und erörtert Möglichkeiten, die Rolle der OSZE bei der Bekämpfung des Antisemitismus zu stärken.

\section{Schlagworte}

OSZE, BDIMR/ODIHR, Antisemitismus, Terrorismus, Sicherheit jüdischer Gemeinden

Bitte zitieren als: Rabbi Andrew Baker, Die OSZE und der Kampf gegen den Antisemitismus in Europa, OSCE Insights 2 (Baden-Baden: Nomos, 2021),

https://doi.org/10.5771/9783748911630-02

\section{Einleitung}

An einem sonnigen Aprilnachmittag des Jahres 2004 ging ich gemeinsam mit Botschafter Christian Strohal, Direktor des Büros für demokratische Institutionen und Menschenrechte (BDIMR/ ODIHR), ins deutsche Bundeskanzleramt. Die hochrangige Berliner Antisemitismus-Konferenz der OSZE $^{1}$ war gerade erst zu Ende gegangen, und wir waren auf dem Weg zu einem Abschlussemp-

* Persönlicher Beauftragter des Amtierenden OSZE-Vorsitzenden für die Bekämpfung des Antisemitismus

Organisation für Sicherheit und Zusammenarbeit in Europa (OSZE)

bakera@ajc.org fang auf Einladung von Bundeskanzler Gerhard Schröder. Nach einhelliger Meinung war diese Konferenz ein Meilenstein in den Bemühungen, die OSZE dazu zu bringen, das Wiedererstarken des Antisemitismus in Europa endlich zum Thema zu machen.

Der deutsche Außenminister Joschka Fischer und der bulgarische Amtierende OSZE-Vorsitzende, Außenminister Solomon Passy, leiteten die Gespräche, und viele Teilnehmerstaaten waren ebenso hochrangig vertreten. Im Jahr zuvor hatte ich eng mit dem US-Botschafter bei der OSZE, Steve Minikes, zusammengearbeitet, um die Organisation zu überzeugen, eine erste Konferenz auf die Beine zu stellen, die schließlich im Juni 2003 in Wien stattfand. Damit war Antisemitismus zu 
einem wichtigen Thema geworden. $\mathrm{Ob}$ es jedoch weitere Veranstaltungen geben würde, war lange Zeit unklar, bis die deutsche Delegation in der Abschlusssitzung ankündigte, eine zweite Konferenz ausrichten zu wollen.

Nun hatten wir diese Konferenz und die so wichtige Berliner Erklärung ${ }^{2}$, die zum Abschluss der Konferenz veröffentlicht wurde. In der Berliner Erklärung von 2004 wird das ODIHR dazu aufgerufen „antisemitische Vorfälle im OSZERaum genau zu verfolgen und dafür alle verfügbaren verlässlichen Informationen heranzuziehen." ${ }^{3}$ Das ODIHR wird außerdem dazu aufgefordert „im gesamten OSZE-Raum Informationen über bewährte Praktiken zur Verhütung und Bekämpfung des Antisemitismus systematisch zu sammeln und zu verbreiten und die Teilnehmerstaaten auf Ersuchen bei ihren Bemühungen im Kampf gegen den Antisemitismus zu beraten."

Ich fand dieses Ergebnis sehr ermutigend, doch Christian Strohal wirkte unzufrieden. „Uns mehr Aufgaben zu geben ist natürlich einfach“, erklärte er. „Aber werden wir auch die notwendige Unterstützung bekommen, diese umzusetzen?“"

Infolge der Berliner Konferenz und der Erklärung rückte das Problem des Antisemitismus zunehmend ins Zentrum der Aufmerksamkeit, und erstmals wurden Anstrengungen unternommen, ernsthaft dagegen vorzugehen. Die OSZE war dafür in gewisser Hinsicht prädestiniert. Die Bekämpfung des Antisemitismus stand einerseits im Einklang mit dem Mandat der menschlichen Dimension. Die Vereinigten Staaten, die besonders lautstark stärkere Anstrengungen forderten, saßen hier zudem mit europäischen Regierungen an einem Tisch. Die OSZE-Verpflichtungen waren zwar rechtlich nicht einklagbar, wurden aber nach ihrer Verabschiedung von den Teilnehmerstaaten, in denen sich das Problem besonders akut darstellte, durchaus ernst genommen. Die langwierige interne Debatte um den genauen Wortlaut der Berliner Erklärung zeigte, dass die Staaten verstanden hatten, dass es auf jedes Wort ankam, und man sich in Zukunft auch daran halten würde.

Nur wenige Wochen nach der Berliner OSZE-Konferenz veröffentlichte die Europäische Stelle zur Beobachtung von Rassismus und Fremdenfeindlichkeit (EUMC) ihren ersten Bericht zum Antisemitismus in der Europäischen Union. ${ }^{4}$ Dieser Bericht umfasste zwei Teile. Der erste Teil basierte hauptsächlich auf den begrenzten, aus Ereignismeldungen und Meinungsumfragen verfügbaren Daten, die von den EUMC-Beobachtern in den Teilnehmerstaaten zusammengetragen worden waren. Der zweite Teil war eine Zusammenstellung von Interviews mit führenden Vertreter*innen europäischer jüdischer Gemeinden. Diese Gespräche vermittelten ein alarmierendes Bild und offenbarten ein Ausmaß an Angst, das es so seit Jahrzehnten nicht mehr gegeben hatte. Einige der Gesprächspartner stellten gar die Zukunft des jüdischen Gemeindelebens in Frage und verwiesen dabei auf die Zunahme antisemitischer Vorfälle und die halbherzige Reaktion staatlicher Stellen. Die EUMC selbst gab zudem an, die Arbeit ihrer Beobachter sei dadurch erschwert, dass die meisten ohne eine Definition 
von Antisemitismus arbeiten mussten und die übrigen sich auf ganz unterschiedlichen Definitionen stützten. ${ }^{5}$

In diesem Beitrag blicke ich auf wesentliche Wendepunkte im Kampf gegen den Antisemitismus zurück. Dazu will ich zunächst zwei Aspekte näher betrachten: die Sicherheitsbedenken jüdischer Gemeinden und die Bedeutung einer gemeinsamen Definition von Antisemitismus. Anschließend betrachte ich Finanzierung, Entscheidungsfindung und das Konsensprinzip als Herausforderungen im OSZE-Kontext. Zuletzt zeige ich Möglichkeiten für den neuen OSZE-Vorsitz auf, der OSZE im Kampf gegen den Antisemitismus wieder eine führende Rolle zu geben.

\section{Der Umgang mit den \\ Sicherheitsbedenken jüdischer Gemeinden}

Im Februar 2020 veranstaltete der albanische OSZE-Vorsitz in Tirana eine Konferenz zur Bekämpfung des Antisemitismus im OSZE-Raum. Er folgte damit der vom italienischen Amtierenden Vorsitzenden im Jahr 2018 begründeten und vom slowakischen Vorsitz 2019 fortgeführten Tradition, diese Konferenzen in den ersten Monaten des Jahres abzuhalten. An der gemeinsam mit dem ODIHR organisierten Konferenz in Tirana nahmen auch die Parlamentarische Versammlung der OSZE und der Hohe Kommissar für nationale Minderheiten (HKNM) teil. Zudem kamen Vertreter*innen anderer internationaler Organisationen, OSZE-Teilnehmerstaaten und Vertreter*innen der
Zivilgesellschaft zur Konferenz. Für sie alle brachte die Konferenz nützliche Empfehlungen hervor. Nur zwei Wochen nach der Konferenz organisierte die Parlamentarische Versammlung der OSZE eine Sondersitzung im Rahmen ihrer Wintertagung in Wien, wo die Parlamentarier*innen das Problem des Antisemitismus diskutieren konnten.

Wir waren davon ausgegangen, dass sich das ganze Jahr über weitere Gelegenheiten ergeben würden, die Empfehlungen bei den Ergänzungstreffen zur Menschlichen Dimension, den Sitzungen des Ständigen Rates und den anstehenden Länderbesuchen der Persönlichen Beauftragten aufzugreifen und weiterzuentwickeln. Durch die erneute Beteiligung der Parlamentarischen Versammlung, deren Resolutionen Vorboten der ersten Antisemitismus-Konferenzen der OSZE gewesen waren, sollten die Bemühungen einen weiteren wertvollen Fürsprecher erhalten.

Leider wurden diese Pläne durch die Corona-Pandemie jedoch jäh ausgebremst. Obwohl wichtige Implementierungstreffen zur Menschlichen Dimension immerhin noch virtuell stattfinden konnten, waren die üblichen Side Events und informellen Zusammenkünfte von zivilgesellschaftlichen Organisationen und Teilnehmerstaaten, die inzwischen auch eine bedeutende Komponente sind, unter diesen Umständen nicht mehr möglich. Über eine Zeitspanne von mehreren Jahren, inklusive dem Jahr 2019, zeigen die Daten zu antisemitischen Vorfällen eine Zunahme oder Stagnation. ${ }^{6}$ Einige Schwankungen lassen sich wahrscheinlich auch auf veränderte 
Praktiken in der Berichterstattung und dem Erfassen von Daten zurückführen. Antisemitismus und Verschwörungstheorien, die Juden mit dem Coronavirus in Verbindung bringen, haben sich in allen sozialen Medien weiter verbreitet.

Im Jahr 2009 und den Folgejahren erhielt ich die Möglichkeit, als Persönlicher Beauftragter des Amtierenden OSZE-Vorsitzenden für die Bekämpfung des Antisemitismus im Rahmen mehrerer Länderbesuche das Thema aufzugreifen. ${ }^{7}$ Dazu traf ich mich zuerst mit Führungspersönlichkeiten und Vertreter*innen der jüdischen Gemeinden und wichtigen Organisationen aus der Zivilgesellschaft und anschließend mit den jeweiligen Regierungschefs. Ein wichtiges Element dieser Besuche war, die Sicherheitsbedürfnisse und -bedenken der jüdischen Gemeinschaft und die entsprechenden Reaktionen der Regierung zu beurteilen. Wenngleich die Situation in jedem Land anders war, sahen sich jüdische Gemeinden in Westeuropa vornehmlich mit zwei Arten von Bedrohungen konfrontiert. Sie waren zum Ziel islamistischer Extremisten und deren internationaler Terrorkampagne geworden und hatten zu Recht Angst um ihr Leben. Zudem kam es regelmäßig zu verbalen und physischen Belästigungen, was in Folge das Behagen und das Sicherheitsgefühl im Alltag der Opfer maßgeblich beeinträchtigte.

Ich machte seinerzeit auf das Problem aufmerksam, und die Teilnehmerstaaten erkannten an, dass der Antisemitismus real ist und die Sicherheitsängste begründet sind. Das bedeutete jedoch nicht unbedingt, dass darauf auch angemessen reagiert wurde. Tatsächlich war ich über- rascht und entmutigt, wenn ich bei meinen Treffen mit Regierungsvertreter"innen erfuhr, wie wenig tatsächlich getan wurde, und welche Ausreden dafür bemüht wurden, warum nicht mehr unternommen wurde. Ein Beispiel ist ein Treffen in Den Haag, wo mir mitgeteilt wurde, die Regierung könne kein erweitertes Sicherheitskonzept für Synagogen anbieten, ohne nicht auch Kirchen und Moscheen etwas Gleichwertiges zu bieten, obwohl diese Gebäude der anderen Religionsgemeinschaften nicht mit derartigen Sicherheitsbedrohungen konfrontiert waren. Da der Regierung die Mittel fehlten, um für alle die nötige Sicherheit zu garantieren, wurde nirgendwo für Sicherheit gesorgt. Die jüdischen Gemeinden fühlten sich daher zu Recht im Stich gelassen.

In Belgien erfuhr ich von Regierungsvertreter*innen, die Gebäude der jüdischen Gemeinden seien ihrer Meinung nach in dieselbe Bedrohungsstufe einzuordnen wie jene der israelischen Botschaft. Sie mussten jedoch einräumen, dass ihnen die finanziellen Mittel fehlten, um Synagogen und jüdischen Schulen das Maß an Sicherheit bieten zu können, das sie selbst für notwendig erachteten.

In Dänemark hatte die jüdische Gemeinde von Kopenhagen darum gebeten, zu den Stoßzeiten vor ihrem Gemeindezentrum und ihren Schulen für sichtbare Polizeipräsenz zu sorgen. Diese Bitte wurde jedoch abgewiesen. Als ich Regierungsvertreter"innen darauf ansprach, sagte man mir, es sei unüblich, bewaffnete Polizist*innen vor Gebäuden zu postieren, dies könne zudem die Bevölkerung verunsichern. Diese Überle- 
gungen waren wichtiger als die tatsächlichen Sicherheitsinteressen der jüdischen Gemeinde. Fünf Monate nach meinem Besuch wurde ein unbewaffneter Wachmann bei einem Terroranschlag vor der Kopenhagener Synagoge getötet.

Bei meinem ersten Besuch in Schweden erfuhr ich, dass die jüdische Gemeinde in Stockholm ein Viertel ihres Budgets für Sicherheit ausgab. Während eines Folgebesuchs erklärte man mir, die Regierung habe die Sicherheitsbedenken aufgegriffen und eine fixe Summe für jedes Gebäude einer religiösen Gemeinde zur Verbesserung des Sicherheitsstandards bereitgestellt. Doch obwohl die Stockholmer Gemeinde ihre Schule und die meisten Gemeindeaktivitäten in einem neu errichteten Gebäude in der Innenstadt gebündelt hatte, stand diesem Zentrum für Gemeindeaktivitäten nicht mehr finanzielle Hilfe zu, wie sie auch jede einzelne Kirche oder Synagoge erhielt.

Es gibt noch weitere Beispiele, die alle eines gemeinsam haben: Sie illustrieren die gegenwärtige Kluft zwischen den Sicherheitsbedürfnissen der jüdischen Gemeinden und der begrenzten Unterstützung der jeweiligen Regierung. Wenngleich die OSZE nicht die einzige Organisation war, die dieses Thema aufgriff, verdeutlicht ein kurzer Rückblick auf die gemeinsamen Bemühungen des ODIHR, wichtiger Teilnehmerstaaten und zivilgesellschaftlicher Gruppen zu diesem Problem die besondere Rolle der OSZE in dieser Frage.

Am 19. März 2012 drang ein islamistischer Terrorist in eine jüdische Schule in Toulouse, Frankreich, ein und ermordete brutal einen Rabbiner und drei kleine
Kinder. Diesen Morden waren zwei Vorfälle vorausgegangen, bei denen derselbe Terrorist vier französische Soldaten mit muslimischem Hintergrund erschoss. Bevor der Terrorist identifiziert werden konnte, waren Strafverfolgungsbehörden davon ausgegangen, der Mann habe aus ultranationalistischer Überzeugung gehandelt. In der Öffentlichkeit herrschte große Empörung über die Morde. Als dann jedoch der Name des Täters bekannt wurde und seine wahren Motive deutlich wurden, schwand ein Teil der Solidarität mit der jüdischen Gemeinde.

Die jüdische Gemeinde Frankreichs verfügte über eine gut funktionierende Sicherheitsorganisation, die den Vorfall untersuchte. Die Schulen waren zu Beginn des Tages immer besonders gefährdet, wenn Eltern und Kinder kamen und gingen und selbst sichere Eingangstüren unter Umständen offengelassen wurden. Auf Überwachungskameras war zu erkennen, wie der Terrorist die Schule am selben Morgen, noch vor seinem Angriff, zunächst ausgespäht hatte. Anwohner*innen in der Gegend hatten das verdächtige Verhalten jedoch nicht bemerkt oder es nicht gemeldet. Führende Vertreter*innen der Gemeinde waren unsicher, ob die entsetzlichen Einzelheiten des Angriffs wirklich publik gemacht werden sollten; und doch wurde gerade durch die Details so deutlich, wie begründet ihre Ängste waren.

Als Persönlicher Beauftragter schlug ich dem Amtierenden Vorsitzenden vor, eine Konferenz zu Fragen der Sicherheit jüdischer Gemeinden zu organisieren. Obwohl es Unterstützung für eine solche Veranstaltung gab, dauerte es einige 
Monate, bis alles bereit war. Der ukrainische OSZE-Vorsitz im Jahr 2013 erklärte sich bereit, das Thema der Sicherheit jüdischer Gemeinden in sein Programm aufzunehmen. Die deutsche Bundesregierung bot an, die Konferenz auszurichten, und Bundesinnenminister Hans-Peter Friedrich kündigte eine Keynote-Ansprache an.

Wir richteten einen Planungsstab ein, dem Vertreter*innen maßgeblicher zivilgesellschaftlicher Organisationen (Europäischer Jüdischer Kongress, American Jewish Committee in Berlin, Zentralrat der Juden in Deutschland) ebenso angehörten wie der Direktor der Abteilung Toleranz und Nichtdiskriminierung (TND) des ODIHR sowie deutsche Regierungsvertreter*innen. Die Konferenz fand unter dem Titel „Die Sicherheitsbedürfnisse jüdischer Gemeinden angehen: Probleme und bewährte Vorgehensweisen" im Juni 2013 in Berlin statt. ${ }^{8}$ Bemerkenswert ist, dass es bei der Konferenz gemeinsame Vorträge der Sicherheitsverantwortlichen jüdischer Gemeinden und ihrer Partner aus den jeweiligen Regierungen und Strafverfolgungsbehörden aus vier wichtigen Teilnehmerstaaten gab. Die Redner*innen stellten dabei nicht nur ihre konkreten Probleme dar, sondern erläuterten auch, wie diese Probleme gemeinsam angegangen wurden. Ein Konferenzbericht enthielt detaillierte Empfehlungen.

Im Jahr darauf übernahm Michael Georg Link den Posten des ODIHR-Direktors. Als ehemaliger Staatssekretär im deutschen Auswärtigen Amt wusste er bestens über diese Konferenz, die dort abgegebenen Empfehlungen und die kon- struktive Rolle der Bundesregierung Bescheid. Im November 2014 fand die Berlin+10-Konferenz in der deutschen Hauptstadt statt; Regierungen und $\mathrm{Zi}$ vilgesellschaft hatten hier die Möglichkeit, das Problem des Antisemitismus im OSZE-Raum zehn Jahre nach der Verabschiedung der Berliner Erklärung neu zu bewerten. ${ }^{9}$ In der Schlussbemerkung appellierte der Schweizer OSZE-Vorsitz besonders an die Strafverfolgungsbehörden, „den sehr realen Bedrohungen für die Sicherheit der jüdischen Gemeinden zu begegnen". ${ }^{10}$ Direktor Link legte einen ambitionierten Plan für den Umgang mit Antisemitismus im OSZE-Raum vor.

Das TND des ODIHR erarbeitete einen detaillierten außerbudgetären Vorschlag unter dem Titel Words into Action to Address Anti-Semitism. Die deutsche Bundesregierung sagte Mittel in erheblichem Umfang zu, und im ODIHR wurde zusätzliches Personal eingestellt. Es gab mehrere Treffen mit Expert"innen aus Teilnehmerstaaten und Vertreter*innen jüdischer Gemeinden, die bereits Erfahrungen mit Sicherheitsvorfällen hatten und somit auch die Best Practices darlegen konnten, was im Idealfall von Regierungen zu leisten wäre. Das Ergebnis dieser Arbeit ist eine Publikation mit dem Titel „Antisemitischen Hassverbrechen begegnen - jüdische Gemeinden schützen: Ein Leitfaden." 11

Der Leitfaden, mittlerweile in viele Sprachen übersetzt, bietet Teilnehmerstaaten und Strafverfolgungsbehörden Handlungsanweisungen, um antisemitische Hassverbrechen zu erkennen und Opfern zu unterstützen. Die dem Leitfaden beigefügten Dokumente enthalten 
etwa kurze Erläuterungen zum Judentum und den jüdischen Feiertagen, an denen besondere Wachsamkeit geboten ist. Eine Tabelle zeigt auf, was u.a. Parlamentarier*innen oder religiöse Gemeinden tun können. Vor allem findet sich hier auch eine Arbeitsdefinition von Antisemitismus. Von zentraler Bedeutung ist ebenso, dass der Leitfaden eine offizielle Publikation von OSZE/ODIHR ist. Er greift die Verpflichtungen der Teilnehmerstaaten auf, die in mehreren Ministerratsbeschlüssen zum Ausdruck kommen. Er bietet damit einzelnen Regierungen die Möglichkeit, den bei Schulungen zur Strafverfolgung im eigenen Land einzusetzen. Dies war ebenso ein wesentlicher Bestandteil des Programmplans zu Words into Action, der auch durch die außerbudgetären Beiträge unterstützt wurde.

Die Erarbeitung und Veröffentlichung des Leitfadens war ein Höhepunkt in den Bemühungen der OSZE, den akutesten Herausforderungen für jüdische Gemeinden zu begegnen. Diejenigen im ODIHR, die den Leitfaden entwickelt hatten, waren von einer robusten zweiten Phase ausgegangen, in der seine Anwendung in der Polizeiausbildung in der gesamten OSZE-Region gefördert werden sollte. Allerdings beendete das ODIHR den Vertrag mit dem Expertenteam, das eigens für das Antisemitismus-Projekt Words into Action eingestellt worden war. Stattdessen bemühte sich das ODIHR die verbleibenden außerbudgetären Mittel zugunsten eines neuen, allgemeineren Projekts zum Thema Kampf gegen Intoleranz umzuschichten - der Antisemitismus wäre darin nur ein Teilaspekt gewesen. Als dem Büro mitgeteilt wurde, dass die Mit- tel nicht umgewidmet werden können, wurden zwar mehrere neue Schulungsprogramme angesetzt, an den langfristigen Plänen änderte sich jedoch nichts.

\section{Den Antisemitismus definieren}

Der erste Schritt im Kampf gegen den Antisemitismus besteht darin, ihn zu definieren. Antisemitismus kann eine Form von Rassismus und Fremdenfeindlichkeit sein, er entzieht sich aber auch unseren allgemeinen Annahmen zur Intoleranz. Er ist an Orten mit bedeutenden jüdischen Gemeinden anzutreffen, findet sich aber auch dort, wo fast keine Juden leben. Es spielt dabei kaum eine Rolle, ob Juden, ähnlich wie andere Einwanderergruppen, Neuankömmlinge sind oder schon seit Jahrhunderten in einem Land ansässig sind.

Verschwörungstheorien mögen zwar jeder Logik entbehren - etwa, wenn Juden gleichzeitig für Kommunismus und Kapitalismus verantwortlich sein sollen -, an ihrer Verbreitung ändert das jedoch nichts. Uralte antisemitische Klischees werde ohne Weiteres auf heutige Gegebenheiten umgemünzt. Im Mittelalter waren die Juden für die Pest verantwortlich, heute stehen sie hinter der CoronaPandemie. Früher warf man den Juden vor, Christen umzubringen, um für rituelle Zwecke an deren Blut zu kommen; heute wird mit dieser alten „Ritualmordlegende" die Behandlung der Palästinenser durch Israel beschrieben. Der Holocaust ist gewiss der bestdokumentierte Völkermord der Neuzeit, und doch gibt es Menschen, die den Holocaust selbst 
oder sein Ausmaß leugnen, und zwar nicht, weil sie kein Interesse an historischer Genauigkeit haben, sondern um Holocaust-Überlebenden und ihren Gemeinschaften auch heute noch Schmerzen zuzufügen.

Dass sich dieses komplexe Verständnis von Antisemitismus und seinen vielfältigen Erscheinungsformen auch beschreiben und vermitteln lassen müsse, hatten Teilnehmer*innen der OSZE-Antisemitismus-Konferenzen im Jahr 2003 und 2004 gefordert. Um diese Lücke zu schließen, kamen einige von uns auf Einladung des EUMC-Direktors im Herbst 2004 zusammen, um eine neue umfassende Antisemitismus-Definition zu erarbeiten. Ich nahm in meiner Funktion als Direktor der Abteilung für internationale jüdische Angelegenheiten des American Jewish Committee daran teil. Unserer Arbeitsgruppe umfasste aber auch Vertreter*innen europäischer jüdischer Organisationen, der Europäischen Kommission gegen Rassismus und Intoleranz (ECRI) des Europarates, und der kurz zuvor gegründeten Abteilung TND des ODIHR. Ein Konsens wurde im Januar 2005 erzielt, im März wurden die Ergebnisse dann als EUMC-Arbeitsdefinition von Antisemitismus, wie sie infolgedessen von vielen genannt wurde, veröffentlicht. Dieses Dokument, das als Lehrmittel für Regierungen und Zivilgesellschaft konzipiert war, enthielt Beispiele für Antisemitismus, unter anderem auch mit Bezug zu Israel. Nachdem diese Arbeit abgeschlossen und die EUMC-Arbeitsdefinition von Antisemitismus veröffentlicht war, wurde sie vom ODIHR in die Unterlagen, die für die neuen ODIHR-Poli- zeischulungsprogramme für den Kampf gegen Hassverbrechen zusammengestellt wurden, übernommen.

Die EUMC-Arbeitsdefinition sorgte sowohl bei Befürwortern als auch Kritikern für erhebliche Aufmerksamkeit, weil sie auch Beispiele für Antisemitismus mit Bezug zu Israel beinhaltete. Einige äußerten die Befürchtung, dies könne dazu missbraucht werden, Israel-Kritiker als Antisemiten abzustempeln, obwohl es in der Definition explizit heißt, dass „Kritik an Israel, die mit der an anderen Ländern vergleichbar ist, nicht als antisemitisch betrachtet werden" könne. ${ }^{12}$ Inzwischen haben führende Vertreter*innen jüdischer Gemeinden jedoch darauf hingewiesen, dass Anti-Israel-Demonstrationen häufig in antisemitische Kundgebungen umschlagen und bisweilen auch physische Angriffe auf jüdische Ziele nach sich ziehen, von den Behörden aber als politisch und nicht als Hassverbrechen eingestuft werden. Diese Beispiele seien daher besonders wichtig.

Man hätte wohl erwartet, dass die OSZE und das ODIHR angesichts ihres Engagements bei der Bekämpfung des Antisemitismus eine zentrale Rolle dabei spielen würden, sich für die Verabschiedung und Anwendung der Arbeitsdefinition einzusetzen. Und doch fällt die Bilanz, trotz nennenswerter Aktionen bestimmter Einzelpersonen und einiger Teilnehmerstaaten, gemischt aus.

Mehrere OSZE-Teilnehmerstaaten übernahmen die EUMC-Arbeitsdefinition. Dazu gehörte das Vereinigte Königreich sowie die Vereinigten Staaten. London nahm die Definition in das Ausbildungshandbuch für Polizeischüler 
auf und Washington verwendete die Arbeitsdefinition bei der Ausarbeitung des ersten internationalen Antisemitismus-Berichts des State Department.

Im Jahr 2009 wurde die EUMC von der neuen und größeren Agentur der Europäischen Union für Grundrechte (FRA) abgelöst. Im März desselben Jahres berief ich als Persönlicher Beauftragter der OSZE unter dem griechischen Vorsitz und in Zusammenarbeit mit dem ODIHR einen runden Tisch in Wien ein, an dem führende Vertreter*innen jüdischer Gemeinden aus dem OSZE-Raum teilnahmen. Der gerade erst ernannte Direktor der FRA nahm ebenfalls teil. Jene Vertreter*innen der jüdischen Gemeinden, denen die Arbeitsdefinition wichtig war, fragten ihn, ob und, wenn ja, wie die FRA sich auch weiterhin für deren Verwendung einsetzen werde. Obwohl er einige Jahre später befand, es falle nicht in die Zuständigkeit der FRA, Definitionen von Antisemitismus oder anderen Formen der Intoleranz zu liefern, ließ der Direktor die Teilnehmer*innen bei diesem Treffen wissen, sie könnten die Definition gern auch weiterhin verwenden. Er hielt zwar Wort, so dass die Definition auch in Folge noch auf der FRA-Website $\mathrm{zu}$ finden war, aber im Gegensatz zu anderen Dokumenten, die von der neuen Agentur übernommen worden waren, stand die Arbeitsdefinition von Antisemitismus nun als einziges Dokument mit dem alten EUMC-Logo auf der Website.

Im Jahr 2013 entfernte die FRA die EUMC-Arbeitsdefinition gänzlich von ihrer Website - im Zuge einer größeren Aufräumaktion, wie es hieß, und zur Erinnerung, dass die Agentur nicht für
Definitionen zuständig sei. Die FRA vertrat nun vielmehr die Position, Minderheiten und andere Opfergruppen sollten selbst die Vorurteile definieren, mit denen sie konfrontiert sind. Zudem wurde darauf verwiesen, dass in einer neueren Erhebung der Agentur, in der es um die Wahrnehmungen und Erfahrungen der jüdischen Bevölkerung im Zusammenhang mit dem Antisemitismus in der EU ging, durchaus auch eine Form des Antisemitismus mit Bezug zu Israel als Auswahlmöglichkeit für die Befragten aufgeführt sei. Tatsächlich gehörte diese Option zu der am häufigsten gewählten. ${ }^{13} \mathrm{Ob}$ wohl die EUMC-Arbeitsdefinition inzwischen ein institutionelles "Waisenkind“ war, wurde sie von immer mehr OSZETeilnehmerstaaten angewendet und den Regierungen auch weiterhin als Orientierungshilfe empfohlen, um Antisemitismus in seinem multidimensionalen Wesen zu erkennen.

Bei der Berlin+10-Konferenz der OSZE, die im November 2014 in Berlin stattfand, bezog sich der Amtierende Vorsitzende aus der Schweiz in seiner $\mathrm{Zu}$ sammenfassung der Ergebnisse des Treffens konkret auf die Definition. Er betonte, die Teilnehmerstaaten der OSZE hätten deutlich gemacht, dass die Arbeitsdefinition von Antisemitismus, die im Jahr 2005 von der EUMC verbreitet wurde und von den Beobachtungsorganisationen in verschiedenen OSZE-Teilnehmerstaaten verwendet wird, auch weiterhin ein nützliches Dokument für Regierungen und Zivilgesellschaft ist - vor allem wenn es darum geht zu erklären, wie der Antizionismus häufig dem Antisemitismus als Maske dient und dass jüdische 
Gemeinden oft zur Zielscheibe antiisraelischer Feindseligkeiten werden. ${ }^{14}$

Im Januar 2015 nahm ich an einem Treffen von Sondergesandten in Prag teil. Mehrere Länder, darunter die Tschechische Republik, das Vereinigte Königreich, die Vereinigten Staaten und Deutschland hatten Sonderbeauftragte für Antisemitismusfragen ernannt. Die EU-Kommission hatte erst kurz zuvor einen Koordinator zu diesem Thema bestimmt. Die neue TND-Direktorin des ODIHR saß ebenfalls mit am Tisch. Im Rahmen der breit angelegten Diskussion ergab sich auch ein lebhafter Austausch über die Arbeitsdefinition, wie man sich dafür einsetzen und wie sie angewendet werden könne. Während die Teilnehmer*innen berichteten, was in ihren jeweiligen Ländern und Organisationen getan werde, vernahm ich erstaunt von der TND-Direktorin, das ODIHR könne die Arbeitsdefinition nur dann anwenden, wenn dies von allen OSZE-Teilnehmerstaaten einvernehmlich mitgetragen werde. Wusste sie denn nicht, dass ihre zwei Vorgänger die Arbeitsdefinition bereits in den von ihnen ausgearbeiteten Materialien verwendet hatten? Boten denn die jüngsten Empfehlungen des Amtierenden OSZE-Vorsitzenden nicht Rechtfertigung genug?

Als Deutschland im Jahr 2016 den OSZE-Vorsitz übernahm, erklärte Berlin die Bekämpfung des Antisemitismus zu einer der Prioritäten. Als Persönlicher Beauftragter arbeitete ich eng mit dem Chef der OSZE-Task Force und mit Botschafter Felix Klein, dem Beauftragten der Bundesregierung für jüdisches Leben in Deutschland und den Kampf gegen An- tisemitismus, zusammen. Zudem übernahm die Internationale Allianz für Holocaust-Gedenken (IHRA), die im Mai 2016 in Bukarest zusammenkam und der seinerzeit 31 Länder angehörten, die ursprüngliche EUMC-Arbeitsdefinition von Antisemitismus mit geringfügigen Anpassungen. Die Definition hatte damit wieder ein Zuhause gefunden und war fortan als „IHRA-Arbeitsdefinition“ bekannt. Der deutsche Vorsitz setzte sich zum Ziel, die Übernahme der Arbeitsdefinition durch die OSZE im Rahmen eines Ministerratsbeschlusses zum Ende des Jahres sicherzustellen.

Das ganze Frühjahr und den Sommer 2016 über unternahm Deutschland umfassende Anstrengungen, um sich die Unterstützung einzelner Teilnehmerstaaten zu sichern. Es ließ Botschafter demarchieren und ein Sondertreffen in Berlin einberufen. Es bemühte sich bei allen EUMitgliedstaaten um Unterstützung, damit deren jeweilige Botschafter bei der OSZE in Wien mit einer Stimme sprechen würden. Als uns zu Ohren kam, dass die dänische Regierung noch Vorbehalte hatte, reisten Botschafter Klein und ich eigens nach Kopenhagen. Wir vermuteten, die dortige Regierung hätte Probleme mit den auf Israel bezogenen Beispielen aus der Arbeitsdefinition, und überlegten gemeinsam, wie wir darauf reagieren sollten. Doch wir hatten uns geirrt. Das dänische Außenministerium wollte sich lediglich vergewissern, dass Deutschland diesen Beschluss wirklich wollte und, dass der Amtierende Vorsitzende meinte, was er sagte. (Und das tat er.) Sobald wir das bestätigt hatten, war Dänemark mit an Bord. 
Als die Kampagne schon eine ganze Zeit lief, fiel uns auf, dass bis dahin noch niemand eine Beschlussvorlage formuliert hatte. Niemand wollte die gesamte Definition für mögliche Zusätze und Streichungen der einzelnen Teilnehmerstaaten öffnen. Eine entsprechende Beschlussvorlage wurde schließlich Anfang September vorgelegt, darin war lediglich die Rede davon, die IHRA-Arbeitsdefinition mit einem Link zur vollständigen Definition auf der IHRA-Website zu übernehmen. So wäre sichergestellt, dass der Wortlaut der Definition und die dazugehörenden Beispiele nicht angetastet werden konnten. Das Implementierungstreffen der Menschlichen Dimension rückte inzwischen näher und wir waren optimistisch, eine Konsenslösung zu erreichen.

Wir hatten jedoch die Russische Föderation nicht bedacht, die bis dahin unser Anliegen nicht mitgetragen hatte. Also traf ich mich beim Implementierungstreffen zur Menschlichen Dimension mit der russischen Delegation, um deren Einwände anzuhören. Sie äußerten zwei Bedenken mit Blick auf die Beschlussvorlage. Erstens lehnten sie einen Beschluss $\mathrm{ab}$, der eine Definition der IHRA billigte, da Russland selbst nicht Mitglied der IHRA war. (Auch viele andere OSZETeilnehmerstaaten waren nicht Mitglied der IHRA, was sie jedoch nicht davon abhielt, das Anliegen zu unterstützen.) Zweitens seien ihre eigenen jüdischen Expert"innen in Russland mit der bestehenden Definition nicht einverstanden, so ihre Erklärung. (Ich hatte bis dahin selbst nichts von führenden jüdischen Vertreter*innen aus Russland gehört). Ich frag- te, ob das ihre einzigen beiden Probleme seien. Ihre Antwort lautete: „Ja, vorerst schon". Es fiel schwer, daraus nicht zu schließen, dass, wenn ein neues Problem notwendig wäre, sie auch eines finden würden. Im Oktober organisierte der Russische Jüdische Kongress in Moskau eine internationale Konferenz. In dem Wissen um die anstehende Entscheidung bei der OSZE verabschiedete die Konferenz eine Resolution, in der sich führende Repräsentanten der russischen jüdischen Gemeinden unmissverständlich hinter die Annahme der Arbeitsdefinition stellten und damit zumindest eine der beiden Ausreden aus dem Weg räumten. ${ }^{15}$

Als ich im Dezember 2016 am Vorabend des Ministertreffens in Hamburg ankam, bestand jedoch wenig Anlass für Optimismus. Die meisten Beschlussvorlagen waren noch nicht konsensfähig, und der Beschluss zur Annahme der Arbeitsdefinition gehörte auch dazu. Am späten Abend saßen der russische Außenminister Sergej Lavrov und der Amtierende OSZE-Vorsitzende Frank-Walter Steinmeier noch einmal zusammen, um Möglichkeiten für eine Einigung auszuloten. Am nächsten Morgen hörte ich von Botschafter Klein, es gäbe gute Nachrichten. Während die Verhandlungen zu anderen Vorlagen festgefahren waren, schien eine Einigung über eine Unterstützung der Arbeitsdefinition doch denkbar. Wir waren - zumindest einige Stunden lang - bester Dinge. Die zwischen den beiden Ministern erzielte Einigung sah vor, dass Russland den Beschluss unterstützen werde, wenn zwei geringfügige Änderungen vorgenommen würden: die Art der 
Bezugnahme auf die IHRA im Text sowie zusätzliche Formulierungen, wonach die Übernahme der Definition durch die OSZE als erster Schritt auf dem Weg zu einem globalen Konsens zu sehen sei. Diese Änderungen zu akzeptieren, wäre aus unserer Sicht unproblematisch gewesen, und so versammelten wir uns an diesem Morgen zu den Textverhandlungen. Als dann aber die russische Delegation das Wort ergriff, um Änderungen vorzuschlagen, waren es nicht mehr nur zwei, sondern viele - auch grundsätzlich neue Formulierungen und die komplette Streichung des so wichtigen Links zur vollständigen IHRA-Definition. Es war klar, dass ein Kompromiss unter diesen Umständen völlig undenkbar war, und Minister Lavrov selbst saß zu dieser Zeit schon wieder im Flieger nach Moskau. Die Beschlussvorlage wurde zurückgezogen.

Im Jahr 2017 erwog der österreichische Vorsitz einen weiteren Versuch, einen OSZE-Ministerbeschluss zur Annahme der Arbeitsdefinition zu ermöglichen. Im Laufe des Jahres wurde jedoch offenbar, dass wir noch immer in der Sackgasse steckten, in die wir 2016 hineingeraten waren. Schweren Herzens kamen wir zu dem Schluss, dass ein zweiter erfolgloser Anlauf womöglich kontraproduktiv wäre. Trotz der eindeutigen Unterstützung, die wir von einer überwältigenden Mehrheit der Teilnehmerstaaten erfuhren, bestand die Gefahr, dass einige behaupten könnten, wiederholtes Scheitern auf Ministerebene müsse die Anwendung der Definition weiter einschränken.

Die Arbeitsdefinition ist, wie oben erwähnt, im Anhang des OSZE/ODIHR-
Leitfadens enthalten, wie auch in einem Dokument für Entscheidungsträger"innen zu Bildungsangeboten gegen Antisemitismus. ${ }^{16}$ Sie ist jedoch nicht zentraler Bestandteil dieser Dokumente. Allerdings haben andere internationale Gremien und einzelne Regierungen bei der Übernahme der Arbeitsdefinition und der Empfehlung, sie auch anzuwenden, beachtliche Fortschritte erzielt. In einer Erklärung des Europäischen Rates im Jahr 2018 werden die EU-Mitgliedsstaaten aufgefordert, die Definition zu übernehmen. ${ }^{17}$ In seinem ersten Antisemitismusbericht aus dem Jahr 2019 appellierte der Sonderberichterstatter der Vereinten Nationen für Religions- und Weltanschauungsfreiheit an die Mitgliedsstaaten der Vereinten Nationen, die Definition anzuwenden, und druckte sie in voller Länge ab. ${ }^{18}$ Selbst die FRA hat seither die Arbeitsdefinition wieder auf ihre Website gestellt und befragt offiziell alle EU-Mitgliedstaaten, wie sie die Definition in ihren jeweiligen nationalen Aktionsplänen gegen Antisemitismus anwenden.

\section{Institutionelle Herausforderungen der OSZE}

Der konsensbasierte Entscheidungsprozess der Organisation erschwert ein zügiges und entschiedenes Handeln zusätzlich. Dies stellt nicht nur eine Herausforderung und sogar ein Hindernis für die Annahme von Ministerratsbeschlüssen dar. Mitunter kann der Prozess auch denjenigen als Vorwand dienen, die entscheiden könnten, dies aber nicht tun. Ein Amtierender Vorsitzender ist flexibel 
genug, eine Expertenkonferenz zu veranstalten oder die Reise eines Persönlichen Beauftragten zu unterstützen. Leitende Mitarbeiter"innen des ODIHR verfügen über beträchtliche Freiräume, wenn es darum geht, Redner*innen vorzuschlagen oder eine kommentierte Tagesordnung aufzusetzen. Verzögerungen oder Untätigkeit sind jedoch nicht nur auf den Wunsch zurückzuführen, Partner in das Verfahren einzubinden. Manchmal sind sie eine Reaktion auf antizipierte Kritik, die möglicherweise gar nicht eintritt.

Das, was sich als "gängige Praxis“ der OSZE etabliert hat, trägt auch dazu bei, Bemühungen zu verzögern oder zu verwässern. Botschafter in Wien sprechen oft von einem "ganzheitlichen“ Ansatz und bestehen darauf, der Kampf gegen den Antisemitismus müsse Bestandteil umfassenderer Bemühungen zur Bekämpfung der Intoleranz sein. Was auf den ersten Blick eine noble und prinzipientreue Haltung sein mag, macht es jedoch schwieriger, die besonderen Elemente des Antisemitismus und der Gefahren für jüdische Gemeinden zu sehen. Hinzu kommt, dass das legitime Interesse der OSZE an geographischer Ausgewogenheit nicht zwangsläufig zu besseren Konferenzen oder besonders effektiven Länderbesuchen beigetragen hat. Das Problem des Antisemitismus, die Größe und die konkreten Sorgen der jüdischen Gemeinden sowie die Verfügbarkeit anerkannter Expert"innen sind ungleichmäBig über den OSZE-Raum verteilt.

Jeder OSZE-Vorsitz legt selbst seine Prioritäten für sein Jahr im Amt fest. Einige haben dem Kampf gegen den Antisemitismus mehr Aufmerksamkeit ge- widmet als andere, obwohl meiner Erfahrung nach niemand das Thema ignoriert hat. Das Thema findet sich zumindest in den spezifischen Programmen, Expertenkonferenzen und Beschlussvorlagen des jeweiligen Amtierenden Vorsitzenden. Botschafter Strohal brachte die Frage auf, welche Unterstützung das ODIHR benötige, um seine Aufgabe erfüllen zu können. Ich glaube, diese Frage ist inzwischen beantwortet: Es gab und gibt Teilnehmerstaaten, die bereit sind zu helfen.

Doch die Bedeutung der ODIHR-Leitung kann nicht hoch genug eingeschätzt werden. Das Engagement des einen Direktors war womöglich bahnbrechend; die Gleichgültigkeit einer anderen Person hat Bemühungen vereitelt, selbst wenn der Vorsitz mehr wollte. Obwohl wichtige Teilnehmerstaaten ihre Unterstützung für künftige ODIHR-Programme zur Bekämpfung des Antisemitismus zugesagt hatten, blieb die ODIHR-Direktorin bei ihrer Entscheidung, sich nur für das viel umfassendere und allgemeiner aufgestellte neue Words into Action-Programm um außerbudgetäre Mittel zu bemühen. Das Ausscheiden leitender TNDMitarbeiter*innen und die Vakanz der Stelle des Fachberaters für Antisemitismus beim ODIHR im Jahr 2020 behinderten die Anstrengungen der Staaten zusätzlich.

\section{Zukunftsaussichten}

Schwedens Ministerpräsident Stefan Löfven hatte geplant, im Oktober 2020 in Malmö eine internationale Konferenz mit Schwerpunkt auf der Bekämpfung 
des Antisemitismus auszurichten. Dies wäre auch eine Gelegenheit gewesen, den zwanzigsten Jahrestag der Stockholmer Konferenz zu feiern, auf der die IHRA ins Leben gerufen wurde. Im Januar 2020 gab Löfven seine volle Zustimmung für die IHRA-Arbeitsdefinition bekannt, die ebenfalls von den Konferenzteilnehmern diskutiert werden sollte. ${ }^{19}$

Es wurde zudem erwartet, dass die Sicherheitsbedenken der jüdischen Gemeinden thematisiert würden, da sich die jüdische Gemeinde in Malmö seit geraumer Zeit gefährdet sieht. Unterdessen arbeitete die dänische Regierung seit dem Terroranschlag auf die Synagoge im benachbarten Kopenhagen im Jahr 2015 eng mit jüdischen Sicherheitsexpert"innen aus Skandinavien zusammen, um einen neuen, kooperativen Ansatz zu entwickeln, der selbst von früheren Kritikern viel Lob erfahren hat. Ein neues Best-Practice-Modell sollte nun auch anderen Interessenten vorgestellt werden.

Da die Konferenz aufgrund der Corona-Pandemie auf Oktober 2021 verschoben werden musste, fällt sie zufällig in das Jahr des schwedischen OSZE-Vorsitzes. Daraus sollte folgen, dass Schweden den Kampf gegen den Antisemitismus zu einem Schlüsselaspekt in seinem Plan für den Vorsitz macht und darüber hinaus die Möglichkeit erhält, auf die Ressourcen des ODIHR zurückzugreifen. Eine neue ODIHR-Leitung könnte wichtige Unterstützung leisten. Mit der Hilfe anderer Teilnehmerstaaten könnten wir erleben, dass die OSZE wieder eine Führungsrolle im Kampf gegen den Antisemitismus übernimmt.
Der Antisemitismus wird bisweilen als der älteste Hass der Welt bezeichnet. Leider müssen wir auch heute noch etwas dagegen tun.

\section{Notizen}

1 OSCE, Consolidated summary of the OSCE Conference on Anti-Semitism in Berlin, 27 July 2004, https://www.osce.or g/cio/35389.

2 OSZE, Berliner Erklärung, 2004, 29. April 2004, https://www.osce.org/files /f/documents/9/8/31432.pdf.

3 Ebenda, S. 2.

4 European Monitoring Centre on Racism and Xenophobia (EUMC), Manifestations of Antisemitism in the EU 2002-2003, 3 May 2004, https://fra.euro pa.eu/sites/default/files/fra_uploads/184 -AS-Main-report.pdf.

5 Ebenda, S. 242.

European Union Agency for Fundamental Rights (FRA), Antisemitism: Overview of data available in the European Union 2008-2018, November 2019, https://fra.europa.eu/sites/default/files/fra _uploads/fra-2019-antisemitism-overview -2008-2018_en.pdf.

OSCE, Country Reports - Personal Representative of the Chairperson-in-Office on Combating Anti-Semitism, https://w ww.osce.org/chairmanship/333786.

OSCE/ODIHR, Summary Report of the Expert Conference on Addressing the Security Needs of the Jewish Community in the OSCE Region: Challenges and Good Practices, 13 June 2013, https://w ww.osce.org/files/f/documents/5/c/10525 3.pdf.

9 OSCE, 10th Anniversary of the OSCE's Berlin Conference on Anti-Semitism: High-Level Commemorative Event and Civil Society Forum, 12-13 November 
2014, https://www.osce.org/odihr/1261 68.

10 OSCE, 10th Anniversary of the OSCE's Berlin Conference on Anti-Semitism: High-Level Commemorative Event, Swiss OSCE Chairmanship conclusions, 12-13 November 2014, https://www.osce .org/files/f/documents/9/0/126710.pdf.

11 OSCE/ODIHR, Understanding AntiSemitic Hate Crimes and Addressing the Security Needs of Jewish Communities: A Practical Guide, 15 May 2017, https:// www.osce.org/odihr/317166.

12 International Holocaust Remembrance Alliance (IHRA), Working Definition of Antisemitism, https://www.holocaustrem embrance.com/working-definition-antise mitism.

13 FRA, FRA survey of Jewish people's experiences and perceptions of antisemitism, 23 March 2012, https://fra.eu ropa.eu/en/publication/2012/fra-survey-je wish-peoples-experiences-and-perceptions -antisemitism.

14 OSCE, oben zitiert (Anmerkung 10).

15 Moscow International Conference on Combating Anti-Semitism, Declaration of the First Moscow International Conference on Combating Anti-Semitism (Moscow Declaration), 2 November 2016, http://mcca.ru/en/news/16.

16 OSCE/United Nations Educational, Scientific and Cultural Organisation (UNESCO), Addressing Anti-Semitism through Education: Guidelines for Policymakers, 31 May 2018, S. 85, https://ww w.osce.org/odihr/383089.

17 Council of the European Union, Fight against antisemitism: Council declaration, 6 December 2018, https://www.co nsilium.europa.eu/en/press/press-releases /2018/12/06/fight-against-antisemitism-co uncil-declaration/.

18 United Nations, Report of the Special Rapporteur on freedom of religion or belief, 20 September 2019, S. 21, https://un docs.org/A/74/358.

19 Government Offices of Sweden, Swedish Prime Minister calls on world to fight for memory of the Holocaust, 22 January 2020, https:/www.government.se/opinio n-pieces/2020/01/opinion-piece/. 
